\title{
Exposição das Equipes de Enfermagem aos Riscos Ocupacionais Causados por Resíduos Biológicos no Hospital Universitário em São Luís do Maranhão, Brasil
}

\section{Exposure of Nursing Teams to Occupational Risks Caused by Biological Waste in University Hospitals in São Luís do Maranhão, Brasil}

\author{
Helone Eloísa Frazão Guimarães Faray*ab; Everton Ferreira Lemos ${ }^{\mathrm{c}}$; Rosemary Matias ${ }^{\mathrm{a}}$; Arlindo Faray Vieira \\ Eduardo de Castro Ferreira ${ }^{\text {ae }}$
}
anniversidade Anhanguera Uniderp, Programa de Pós-Graduação Stricto Sensu em Meio Ambiente e Desenvolvimento Regional MS, Brasil

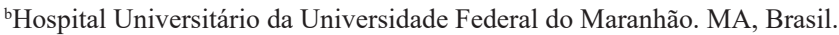
'Universidade Federal do Mato Grosso do Sul. MS, Brasil.

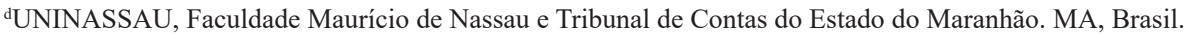
'Fundação Oswaldo Cruz. MS, Brasil.
*E-mail: helonefaray@hotmail.com

\begin{abstract}
Resumo
As atividades de trabalho dos profissionais de enfermagem estão sujeitas a riscos e situações geradoras de desgaste do profissional, contribuindo para ocorrência de doenças e contaminação do ambiente. Objetivou-se analisar os riscos ocupacionais relacionados aos resíduos biológicos hospitalares presentes no cotidiano das equipes de enfermagem dos diferentes setores hospitalares do cuidado à criança, associado ao tempo de serviço. Trata-se de uma pesquisa de natureza aplicada com abordagem quantitativa de caráter descritivo exploratório, transversal, realizada no Hospital Universitário em São Luís do Maranhão, onde a equipe de enfermagem é composta por enfermeiros, técnicos e auxiliares de enfermagem. Foi aplicado questionários a 98 profissionais de enfermagem que compõem a unidade de saúde da criança do hospital universitário em São Luís do Maranhão, no ano de 2018. Em relação aos acidentes de trabalho sofridos pelos profissionais que possuem menos de cinco anos de serviço, a categoria enfermeiro apresentou maior percentual (31,6\%), seguido pelo técnico de enfermagem (21,6\%). A maioria dos trabalhadores $(75,6 \%)$ que relataram ter recebido treinamento em serviço não se acidentaram. Medidas preventivas e estratégias de educação em saúde devem ser assimiladas entre profissionais de enfermagem, sobre o manejo adequado de resíduos e incentivar a implementação de precauções para minimizar a ocorrência de agravos que comprometem a saúde e atividades laborais desenvolvidas no seu ambiente, bem como orientações sobre as consequências dos acidentes de trabalho.
\end{abstract}

Palavras-chave: Acidentes de Trabalho. Enfermagem. Ambiente Hospitalar.

\begin{abstract}
The work activities of nursing professionals are subject to risks and situations that generate professional wear, contributing to the occurrence of diseases and the environment contamination. The objective was to analyze the occupational risks related to hospital biological waste present in the daily lives of nursing teams from different childcare sectors hospital. It is a research of an applied nature with a quantitative approach of an exploratory, cross-sectional descriptive character, carried out at the University Hospital in São Luis do Maranhão, where the nursing team is composed of nurses, technicians and nursing assistants. Questionnaires were applied to 98 nursing professionals who made up the child health unit of the university hospital in São Luís do Maranhão, in the year 2018. Regarding occupational accidents suffered by professionals who have less than five years of service, the category of nurse presented a higher percentage (31.6\%) followed by a nursing technician (21.6\%). Most workers (75.6\%) who reported having received on-the-job training did not have an accident. Preventive measures and health education strategies must be assimilated among nursing professionals, regarding the adequate waste management and encourage the precautions implementation to minimize the occurrence of injuries that compromise health and work activities carried out in their environment, as well as guidance on the consequences of accidents at work.
\end{abstract}

Keywords: Accidents at Work. Nursing. Hospital Environment.

\section{Introdução}

O risco, segundo a Agência Nacional de Vigilância em Saúde, Anvisa (2017), é definido como uma ou mais condições de uma variável com potencial necessário para causar danos. Esses danos podem ser entendidos como lesões a pessoas, danos a equipamentos e instalações, danos ao meio ambiente, perda de material em processo, ou redução da capacidade de produção.

A presença dos riscos ocupacionais na execução das atividades laborais dos profissionais de saúde oferece uma visibilidade multifatorial, devido à diferença dos fatores de riscos a que estão sujeitos, dependendo da atividade realizada (DUARTE; MAURO, 2010).
Entre os trabalhadores de saúde, a enfermagem, é a categoria que mais entra em contato físico com os doentes, prestando assistência ininterrupta, 24 horas por dia e executora de cerca de $60 \%$ das ações de saúde (CARVALHO; MAGALHÃES, 2013).

O principal risco ocupacional que afeta a saúde dos profissionais de enfermagem é a exposição a material biológico, devido à possibilidade de transmissão de patógenos, entre eles o vírus Imunodeficiência Humana (HIV) e das Hepatites virais (ARAÚJO et al., 2012).

Estudos sobre os riscos de acidentes com material biológico em profissionais de enfermagem no ambiente 
hospitalar, destacam que as principais causas dos acidentes são o reencape de agulhas e a não adesão às medidas preventivas, incluindo como fatores condicionantes a sobrecarga de trabalho (RODRIGUES et al., 2019; JANUÁRIO et al., 2017; BARROS et al., 2016)

Desta forma, o presente estudo teve como objetivo analisar os riscos ocupacionais relacionados aos resíduos biológicos hospitalares presentes no cotidiano das equipes de enfermagem associado ao tempo de serviço, nos diferentes setores hospitalares do cuidado à criança do Hospital Universitário em São Luís do Maranhão, Brasil.

\section{Material e Métodos}

\subsection{Local e tipo de estudo}

Trata-se de uma pesquisa de natureza aplicada com abordagem quantitativa de caráter descritivo exploratório, transversal, realizada no Hospital Universitário em São Luís do Maranhão nas dependências da unidade de atenção a criança (internação pediátrica) composta por três alas: Ala A, onde se encontram internados os pacientes cirúrgicos, Ala D, os pacientes com doenças infectocontagiosas, e Ala E, os pacientes com patologias diversas além da UTI pediátrica (UTI ped).

A escolha dessa população atende aos objetivos da pesquisa, uma vez que está diretamente envolvida com o manejo dos Resíduos Sólidos dos Serviços de Saúde (RSSS), por ser a categoria mais numerosa no conjunto dos trabalhadores da saúde e, consequentemente, aquela que mais executa procedimentos hospitalares e tende a produzir maior quantidade de resíduos. Foi escolhida toda a equipe de enfermagem composta por enfermeiros, técnicos e auxiliares de enfermagem da unidade de atenção a criança que é composta pelo setor da internação pediátrica e UTI pediátrica.

Quanto a equipe de enfermagem, 23 (23,5\%) são enfermeiros, $63(64,3 \%)$ técnicos em enfermagem e 12 (12,2\%) auxiliares de enfermagem, totalizando uma população de 131 profissionais de enfermagem, dos quais 98 participaram da pesquisa de acordo com os critérios de inclusão.

\subsection{Critérios de inclusão}

Os critérios de inclusão foram os seguintes: atuar na unidade de atenção a criança (ALA A, ALA D, ALA E e UTI PED), estar presente no setor no dia da entrega do instrumento de coleta de dados, participar voluntariamente da pesquisa e assinar o Termo de Consentimento Livre e Esclarecido, após lerem os objetivos da pesquisa, etapa necessária para o início da entrevista.

A pesquisa atendeu aos preceitos e recomendações da Resolução $n^{\circ} 466 / 2012$, que determina as diretrizes e normas regulamentadoras de pesquisas envolvendo seres humanos, garantindo o sigilo, anonimato e a confidencialidade dos participantes.

A pesquisa foi aprovada pelo Comitê de Ética em Pesquisa da UFMA, Comissão Científica-COMIC-HU-UFMA, nº 48/2018.

\subsection{Coleta de dados e sujeitos da pesquisa}

Foram coletados dados primários e secundários. Os primários foram colhidos juntos aos profissionais de enfermagem, por meio de questionário composto por dez questões, sendo perguntas abertas e fechadas. Integraram a pesquisa todos os profissionais do setor de atenção à saúde da criança que compõem a equipe de enfermagem e que aceitaram participar. A coleta de dados foi realizada nos meses de julho a setembro do ano de 2018 .

\subsection{Tratamento dos dados e análise estatística}

Os dados foram tabulados no Microsoft Office Excel versão 2016. O programa utilizado para análise foi o EPIINFO versão 7 (Centers for Diseases Control and Prevention, Atlanta/Geórgia/Estados Unidos). Para verificar possíveis associações entre as variáveis de estudo foi utilizado o teste Qui-quadrado, com os respectivos intervalos de confiança de 95. Foram apresentados descritivamente por meio de representação tabular constando de frequência absoluta, relativa e o valor de $p$ das associações.

\section{Resultados e Discussão}

Foram avaliados 98 profissionais que atuam na enfermagem, cuja maioria $(64,3 \%)$ eram Técnicos de Enfermagem. Um total de $54(55,1 \%)$ profissionais tinham tempo de serviço entre um e cinco anos, identificou-se que, a maioria da equipe de enfermagem $(83,7 \%)$ está há mais de um ano atuando no serviço. Quanto ao setor de trabalho, na UTI Pediátrica $(40,8 \%)$ apresentaram-se com a maioria de servidores entrevistados, seguidos das unidades de Ala E $(23,5 \%)$, Ala A (20,4\%) e a Ala D (15,3\%) (Quadro 1).

Quadro 1 - Número e porcentagem de profissionais de enfermagem segundo a sua função, tempo de serviço e local de trabalho no Hospital Universitário de São Luís do Maranhão - 2018, (N.98)

\begin{tabular}{|c|c|c|}
\hline Variáveis & $\mathbf{N}$ & $\%$ \\
\hline \multicolumn{3}{|c|}{ Qual a sua função no hospital? } \\
\hline Auxiliar de enfermagem & 12 & 12,2 \\
\hline \begin{tabular}{|l|} 
Enfermeiro \\
\end{tabular} & 23 & 23,5 \\
\hline Técnico de enfermagem & 63 & 64,3 \\
\hline Total & 98 & 100 \\
\hline \multicolumn{3}{|l|}{ Qual o seu tempo de serviço? } \\
\hline Entre um e cinco anos & 54 & 55,1 \\
\hline Mais de cinco anos & 28 & 28,6 \\
\hline Menos de um ano & 16 & 16,3 \\
\hline Total & 98 & 100 \\
\hline \multicolumn{3}{|l|}{ Qual o seu setor de trabalho? } \\
\hline Ala A - Cirúrgica & 20 & 20,4 \\
\hline Ala D - Infecto-contagiosas & 15 & 15,3 \\
\hline Ala E-Patologias & 23 & 23,5 \\
\hline UTI PED - Pediátrica & 40 & 40,8 \\
\hline Total & 98 & 100 \\
\hline
\end{tabular}

Fonte: Dados da pesquisa.

Para a análise de associação entre as variáveis tempo de serviço ( $<5$ anos e $\geq 5$ anos) e ter sofrido um acidente de trabalho no hospital universitário conforme a categoria 
profissional, este estudo identificou que houve associação entre o tempo de serviço $<5$ anos com acidentes para as categorias Enfermeiro e técnico em Enfermagem $(p<0,001)$. A categoria Enfermeiro, com tempo $<5$ anos, apresentou percentual de acidente maior quando comparados com os Técnicos de Enfermagem (31,6\% versus 21,6\%) (Quadro 2).

Quadro 2 - Número e porcentagem de profissionais de enfermagem segundo sua categoria profissional e tempo de serviço relacionado com o acidente de trabalho no Hospital Universitário de São Luís do Maranhão - 2018 (N=98)

\begin{tabular}{|l|c|c|c|}
\hline \multirow{2}{*}{\multicolumn{1}{|c|}{ Categoria }} & \multicolumn{3}{c|}{ Acidente de Trabalho } \\
\cline { 2 - 4 } & Sim (N/\%) & Não (N/\%) & p Valor \\
\hline Enfermeiro & & & \\
\hline$<5$ anos & $6(31,6 \%)$ & $13(68,4 \%)$ & \multirow{2}{*}{$<0,001$} \\
\hline$\geq 5$ anos & $1(25,0 \%)$ & $3(75,0 \%)$ & \\
\hline Técnico de Enfermagem & & & \\
\hline$<5$ anos & $11(21,6 \%)$ & $40(78,4 \%)$ & \multirow{2}{*}{$<0,001$} \\
\hline$\geq 5$ anos & $3(25,0 \%)$ & $9(75,0 \%)$ & \\
\hline Auxiliar de Enfermagem & & & \\
\hline$<5$ anos & $0(0,0 \%)$ & $0(0,0 \%)$ & \multirow{2}{*}{-} \\
\hline$\geq 5$ anos & $4(33,3 \%)$ & $8(66,7 \%)$ & \\
\hline
\end{tabular}

Nota: Teste Qui quadrado com $\mathrm{p}<0.05$.

Fonte: Dados da pesquisa.

Em estudo realizado com acidentes de trabalho e profissionais da saúde foi afirmado que a quantidade de registros de acidentes de trabalho é maior entre os profissionais de enfermagem (auxiliar, técnico e enfermeiro), em relação aos outros profissionais, chegando a 73,6\% das ocorrências. Este fato reforça a preocupação evidenciada pela literatura sobre acidentes de trabalho entre profissionais desta área por estarem mais expostos a riscos ocupacionais devido às peculiaridades das atividades de cuidados diretos e ininterruptos aos pacientes (BAKKE; ARAÚJO, 2010).

De acordo com Ribeiro e Shimizu (2007) dos acidentes com trabalhadores de enfermagem, constatou-se que, 92\% dos acidentes de trabalho são gerados por más condições de trabalho, cargas no desenvolvimento do processo de trabalho da enfermagem, desconhecimento de medidas preventivas, entre outras.

Entre os tipos de acidentes, os perfurocortantes são caracterizados como principal tipo de acidente na enfermagem (MALAGUTI-TOFFANO et al., 2015). Entre os perfurocortantes, estão os bisturi, com descarte inadequado e o reencape de agulha, associados a práticas que contrariam as recomendações oficiais de biossegurança (RODRIGUES et al., 2017; DORNELLES et al., 2016).

No Quadro 3, a análise sobre a associação entre profissionais de enfermagem que receberam treinamento sobre biossegurança relacionado com acidente de trabalho no hospital agrupados de acordo com tempo de serviço no Hospital Universitário de São Luís do Maranhão, mostrou que a maioria dos servidores que relataram ter recebido treinamento de biossegurança no serviço não se acidentaram ( $<5$ anos, 75,6\%; $\geq 5$ anos, 76,5\%). Para os servidores com tempo de serviço $<5$ anos, houve associação entre ter recebido treinamento de biossegurança com o acidente de trabalho $(\mathrm{p}<0,05)$. Isto é, foi observado que o treinamento contribui para a redução de acidentes. Não houve associação entre o tempo de serviço $\geq 5$ anos.

Quadro 3 - Número e porcentagem de profissionais de

enfermagem que receberam treinamento sobre biossegurança e se acidentaram no hospital relacionado ao tempo de serviço no Hospital Universitário de São Luís do Maranhão - 2018, (N.98)

\begin{tabular}{|c|c|c|c|c|c|c|}
\hline Treinamento & Acidente d & $\begin{array}{l}\text { alho de acor } \\
\text { serviço }\end{array}$ & tempo de & & & \\
\hline & & $<5$ anos & & & $\geq 5$ anos & \\
\hline & Sim N (\%) & Não N (\%) & pValor & Sim N(\%) & Não N(\%) & pValor \\
\hline Sim & $12(24,4 \%)$ & $37(75,6 \%)$ & \multirow{2}{*}{$<0,001$} & $4(23,5 \%)$ & $13(76,5 \%)$ & \multirow{2}{*}{0,093} \\
\hline Não & $5(23,8 \%)$ & $16(76,1 \%)$ & & $4(36,3 \%)$ & $7(63,7 \%)$ & \\
\hline
\end{tabular}

Nota: Teste Qui quadrado com $\mathrm{p}<0.05$.

Fonte: Dados da pesquisa.

Silva et al. (2011) destacam que os acidentes com materiais perfurocortantes expondo os trabalhadores a material biológico são problemas frequentes vivenciados pela equipe de enfermagem no ambiente hospitalar. Isto se deve ao fato de grande parte das atividades dos trabalhadores de enfermagem estar concentrada na administração de medicamentos e soroterapia, atividades que envolvem a manipulação constante de dispositivos perfurocortantes.

Portanto, todos os profissionais que compõem a equipe de enfermagem estão suscetíveis a sofrer acidentes. Os trabalhadores de saúde jamais devem se afastar do cumprimento das práticas de biossegurança e conhecimento a respeito da ocorrência dos acidentes, como o uso dos Equipamentos de Proteção Individual (EPI), que lhes oferecem maior segurança para o desenvolvimento de suas atividades (SILVA et al., 2012).

Observa-se a importância do treinamento em serviço, da educação permanente para os profissionais da saúde, pois um maior conhecimento por parte desses profissionais pode conduzir a prevenção de acidentes de trabalho. A educação em saúde é um processo pedagógico e político que visa o desenvolvimento reflexivo e critico permitindo apontar a realidade e propor ações inovadoras capaz de opinar nas decisões no ato de cuidar de si e do próximo de maneira 
correta e com qualidade (MACHADO et al., 2007).

\section{Conclusão}

Identificou-se que os acidentes de trabalho que ocorreram com menos de 5 anos de serviço foram mais frequentes para as categorias enfermeiro e técnico de enfermagem. A categoria enfermeiro apresentou maior frequência de acidentes de trabalho que a categoria técnico de enfermagem. Percebe-se que a maioria dos trabalhadores que relataram ter recebido treinamento em serviço, como conhecimento sobre biossegurança, não se acidentaram, evidenciando a importância da educação continuada dirigida aos trabalhadores da saúde, sendo visualizada pelos profissionais na forma de treinamento em serviço. Medidas preventivas e estratégias de educação em saúde devem ser assimiladas entre profissionais de enfermagem, sobre o manejo adequado de resíduos e incentivar a implementação de precauções para minimizar a ocorrência de acidentes.

\section{Referências}

ANVISA. Agencia Nacional de Vigilância Sanitária. Segurança no ambiente hospitalar. 2017. Disponível em: <http://portal.anvisa. gov.br/documents/33852/271855/Seguran \%C3\%A7a+no+am biente+hospitalar/473c5e32-025a-4dc2-ab2e-fb5905d7233a>. Acesso em: 15 ago. 2019.

ARAÚJO, T M. et al. Acidente ocupacional e contaminação pelo HIV: sentimentos vivenciados pelos profissionais de enfermagem. Rev. Pesq. Cuidado Fund., v.4, n.4, p.2972-2979,2012.

BAKKE, H.A.; ARAÚJO, N. M. C. Acidentes de trabalho com profissionais de saúde de um hospital universitário. Rev. Produção, v.20, n.4, p.669-676, 2010. doi: 10.1590/S010365132010005000015.

BARROS, D.X. et al. Análise de 10 anos de acidentes com material biológico entre a equipe de enfermagem. Rev. Eletr. Enferm., v.18, n.4, 2016. doi:10.5216/ree.v18.35493

CARVALHO, C.; MAGALHÃES, S. R. Principais fatores que interferem na saúde dos profissionais de enfermagem, uma visão biopsicossocial. J. Res. Fundamental Care On Line, v.5, n.3, p.122-131, 2013.

DUARTE, N.S.; MAURO, M.Y.C. Análise dos fatores de riscos ocupacionais do trabalho de enfermagem sob a ótica dos enfermeiros. Rev. Bras. Saúde Ocupac., v.35, n.121, p.157-167, 2010 .

DORNELLES, C. et al. Exposição de profissionais de saúde ao material biológico: estudo no ambiente hospitalar. J. Nurs. Health, v.6, n.1, p.64-75, 2016.

JANUÁRIO, G. C. et al. Acidentes ocupacionais com material potencialmente contaminado envolvendo trabalhadores de enfermagem. Cogitare Enferm., v. 22, n. 1, p. 1-9, 2017.

MACHADO, M. F. A. S et al. Integralidade, formação de saúde, educação em saúde e as propostas do SUS: uma revisão conceitual. Ciênc. Saúde Coletiva, v.12, n.2, p.335- 342,2007. doi: 10.1590/S1413-81232007000200009.

MALAGUTI-TOFFANO, S.E. et al. Adesão às precauçõespadrão entre profissionais da enfermagem expostos a material biológico. Rev. Eletr. Enferm., v.17, n.1, p.131-135, 2015. doi: 10.5216/ree.v17i1.29269.

RIBEIRO, E.J.G.; SHIMIZU, H.E. Acidentes de trabalho com trabalhadores de enfermagem. Rev. Bras. Enferm., v.60, n.5, p.535-540, 2007. doi: 10.1590/S0034-71672007000500010

RODRIGUES, P.P; DE ALENCAR, R.M. Riscos de acidentes com material biológicos em profissionais de enfermagem no ambiente hospitalar: revisão integrativa. Rev. Ciênc. Saúde Nova Esperança, v.17, n.2, p.64-72, 2019.

RODRIGUES, P.S. et al. Acidente ocupacional entre profissionais de enfermagem atuantes em setores críticos de um pronto-socorro. Escola Anna Nery, v.21, n.2, 2017.

SILVA, D.D.S.D. et al. Depressão e risco de suicídio entre profissionais de Enfermagem: revisão integrativa. Rev. Esc. Enferm. USP, v.49, n.6, p. 1023-1031, 2011.

SILVA, M.; CORTEZ, E.; VALENTE, G. S. C. Acidentes com materiais perfurocortantes e biológicos no ambiente hospitalar: análise da exposição ao risco e medidas preventivas. Rev Pesq. Cuidado Fundamental, v.3, n.2, p.1856-1872, 2012. 